Article

\title{
Dynamical Stability and Geometrical Diagnostic of the Power Law K-Essence Dark Energy Model with Interaction
}

\author{
Bo-Hai Chen ${ }^{1,2}$, Ya-Bo Wu ${ }^{1, *}$, Dong-Fang Xu ${ }^{1}$, Wei Dong ${ }^{1}$ and Nan Zhang ${ }^{1,3}$ \\ 1 Department of Physics, Liaoning Normal University, Dalian 116029, China; \\ bohai_chen@nciae.edu.cn (B.-H.C.); xdf9678@163.com (D.-F.X.); dongweiooooo@163.com (W.D.); \\ zhnan@ustc.edu.cn (N.Z.) \\ 2 School of Liberal Arts and Sciences, North China Institute of Aerospace Engineering, \\ Langfang 065000, China \\ 3 Peng Huanwu Center for Fundamental Theory, Hefei 230026, China \\ * Correspondence: ybwu61@163.com
}

Received: 21 October 2020; Accepted: 13 December 2020; Published: 18 December 2020

check for updates

\begin{abstract}
We investigate the cosmological evolution of the power law k-essence dark energy (DE) model with interaction in FRWL spacetime with the Lagrangian that contains a kinetic function $F(X)=-\sqrt{X}+X$. Concretely, the cosmological evolution in this model are discussed by the autonomous dynamical system and its critical points, together with the corresponding cosmological quantities, such as $\Omega_{\phi}, w_{\phi}, c_{s}^{2}$, and $q$, are calculated at each critical point. The evolutionary trajectories are drawn in order to show the dynamical process on the phases plan around the critical points. The result that we obtained indicates that there are four dynamical attractors, and all of them correspond to an accelerating expansion of universe for certain potential parameter and coupling parameter. Besides that, the geometrical diagnostic by the statefinder hierarchy $S_{3}^{(1)}$ and $S_{4}^{(1)}$ of this scalar field model are numerically obtained by the phase components, as an extended null diagnostic for the cosmological constant. This diagnostic shows that both the potential parameter $\lambda$ and interaction parameter $\alpha$ play important roles in the evolution of the statefinder hierarchy.
\end{abstract}

Keywords: k-essence dark energy; dynamical stability; statefinder hierarchy

\section{Introduction}

There are two important stages in cosmology, the early inflation and the late time accelerated expansion. The inflation that we postulate is to explain some issues, such as the flat problem and the horizon problem, etc. While, the accelerating expansion is based on the observations on the luminosity-redshift relation of distant Ia supernovas [1,2], Cosmic Microwave Background [3], and Baryon Acoustic Oscillations [4], which indicate that the current energy density in the universe is composed by $68.3 \%$ dark energy (DE), $26.8 \%$ dark matter (DM), and $4.9 \%$ baryons [5] in order to drive the late time acceleration approximately. More details and cosmic constrain by observation are in [6-9]. Since its first observation in 1998, over the last twenty years, there have been many models to make explanation for the physical mechanism of this phenomenon. Among them, the simplest one is the $\Lambda$ CDM model with a constant equation of state (EoS) $w_{\Lambda}=-1$, which provides the negative pressure for the expansion. $\Lambda \mathrm{CDM}$ model is in good accordance with the observation, but it has some crucial problems, such as the cosmological constant problem, the age problem [10-14], and the tensions on the parameters $H_{0}$ and $\sigma_{8}$ in the $\Lambda \mathrm{CDM}$ model in recent years [15-20]. Instead of the $\Lambda \mathrm{CDM}$ model, there is a class of phenomenological models with a scalar field to reconcile the problems above; for example, quintessence, phantom, quintom, tachyon, k-essence, and DBI models, etc. Among them, 
the quintessence model is one of the most popular one [21]. Its Lagrangian density $\mathcal{L}=X-V(\phi)$, being the pressure, in the action is described by a single scalar field $\phi$, and a canonical kinetic energy $X \equiv-\frac{1}{2} \partial_{\mu} \phi \partial^{\mu} \phi$. To some extent, the quintessence model can describe a range of EoS necessary for an accelerated expansion, but the single-field quintessence with a canonical kinetic term limits the EoS $w$ to be less than -1 . To this point, the quintom model with multiple fields [22] and some k-essence models with non-canonical kinetic terms are developed in order to realize the crossing of the phantom divide. Additionally, the combination of data sets Plank+R16+JLA support this point, such as in [15], for $w=-1.079_{-0.057}^{+0.072}$.

It is known that the k-essence model is a better substitution for the quintessence model. Although it firstly originated from k-inflation coming from the string theory to explain the early inflation of the universe [23]; fortunately, it could also explain the late time accelerated expansion as DE [24], even in the form as a decent generalization of many scalar field dark energy models. Unlike the canonical kinetics that are defined by the quintessence model, the k-essence model provides a variety of non-canonical terms, i.e., $\mathcal{L}=F(X, \phi)$ [25], which contains higher order terms of $X$. As a result, the k-essence model with non-canonical kinetic term in Largragian can also reconcile the $H_{0}$ tension problem by making the EoS smaller than -1 . The form of $F(X, \phi)$ was first discussed as a purely kinetic function $F(X)$, with a constant potential, $V(\phi)=$ const [26]. Further more, two common forms along with potential are $F(X, \phi)=F(X)-V(\phi)$ and $F(X, \phi)=F(X) V(\phi)$, which were widely investigated in $[27,28]$. Some of the modified kinetic terms were discussed, such as $F(X)=K X+L X^{2}$ [29], $F(X)=\frac{1}{2 \alpha-1}\left((A X)^{\alpha}-2 \alpha \alpha_{0} \sqrt{A X}\right)$ [30], and $F(X)=A \sqrt{X}-B X^{\alpha}$ [31] etc., which belongs to the class of power law k-essence dark energy model with the power law function $F(X)=\sum a_{i} X^{b_{i}}$. The approximation of the potential in scalar field dark energy models are discussed in [32], with both canonical and noncanonical kinetic terms.

In another aspect, from the matter clustering properties, dark matter (DM) and dark energy are not the same substance; however, there are researches regarding the interactions between them, even some nonlinear interaction forms [33-37], which can provide a mechanism for generating acceleration. By the recent observation, the interaction between DM and DE is too little to alleviate the coincidence problem, while, in our work, the k-essence model with the interaction between DM and DE can be a candidate, which helps to explain the $H_{0}$ tension and $\sigma_{8}$ tension between $\mathrm{CMB}$ and structure formation measurements [15-17].

The aim of this paper, which is based on the researches above, is to consider a model with $F(X, \phi)=F(X) V(\phi)-f(\phi)$, where $F(X)=-\sqrt{X}+X, V(\phi) \propto 1 / \phi^{2}$, and $f(\phi)=0$ [38], together with a certain kind of interaction $Q$. We investigate the possible cosmological behavior of this model in Friedmann-Robertson-Walker-Lemaitre (FRWL) spacetime by performing a phase-space and stability analysis. The theory are based on $[39,40]$ judging the stability of the critical points by the eigenvalues; whereas, in this model for the convenience of calculation, it prefers the method by the determinant and trace of the Jacobian matrix of the autonomous differential equations [41]. Some cosmological quantities will be calculated for each critical point, such as the dark energy density parameter $\Omega_{\phi}$, the equation of state (EoS) parameter $w_{\phi}$ of dark energy, the sound speed $c_{S}^{2}$, and the deceleration parameter $q$.

Finally, in order to distinguish this k-essence model from $\Lambda C D M$ model, there are two main kinds of "null measure": the Om diagnostic and the statefinder diagnostic. Om is constructed from the Hubble parameter $H$, and it provides a null test of the $\Lambda$ CDM model [42,43]. In recent years, in order to distinguish those models from the best fitting model, the $\Lambda$ CDM model, the statefinder hierarchy is used, which originate from statefinder diagnostic [44,45]. The statefinder pair $\{r, s\}$, is composed by the scale factor $a(t)$ with its second and third derivatives; however, the statefinder hierarchy is based on the even higher derivatives [46]. In this paper, statefinder hierarchy $S_{3}^{(1)}$ and $S_{4}^{(1)}$ are analyzed to the scalar dark energy model by the phase components (the auxiliary variables) in the autonomous equations, unlike the method that was mentioned before, which depends on the cosmological quantities. It shows that the hierarchies are varied from two parameters $\lambda$ and $\alpha$ by the 
trajectories. This novel method is based on [47-49], which is used for the quintessence model and $I \omega C D M m o d e l$, and it could be generated to a range of scalar DE models in the future.

This paper is organized, as follows: in the following section, we review k-essence dark energy models and its stability analysis. In the third section, we consider the dynamics of the k-essence scalar field with the interaction $Q=\alpha H \rho_{m}$ (the coupling parameter $\alpha$ is a real arbitrary constant). In the fourth section, the statefinder diagnostic and statefinder hierarchy are analyzed in order to distinguish from $\Lambda C D M$ model. Finally, we close with a few concluding remarks in the fifth section.

\section{The Power Law K-Essence Dark Energy Model and Its Stability Analysis}

We consider k-essence dark energy models with Lagrangian

$$
L=p_{\phi}=G(X) U(\phi),
$$

where the kinetic term $G(X)$ and potential term $U(\phi)$ are analytic functions of $X$ and $\phi$, respectively. Throughout this paper, we will work with a flat, homogeneous, and isotropic FRWL spacetime having signature $(-,+,+,+)$ and in units $c=8 \pi G=1$. We are interested in the power law k-essence with a general form of kinetic term $G(X)=-K(\phi) \sqrt{X}+L(\phi) X$, which has been studied in $[29,38,50]$. Hence, the scalar field $\phi$ is redefined as the one in [29]. Consequently, Equation (1) is rewritten as $p_{\phi}=F(X) V(\phi)$, where the new kenetic term $F(X)=-\sqrt{X}+X$ and new potential term $V(\phi)=\left(K^{2} / L\right) U(\phi)$. Subsequently, the corresponding energy density $\rho_{\phi}$, the EoS parameter $w_{\phi}$ and the effective sound speed $c_{\mathrm{s}}^{2}$ are, respectively, given by

$$
\begin{aligned}
\rho_{\phi} & =V(\phi)\left[2 X F_{X}-F\right]=X V \\
w_{\phi} & =\frac{F}{2 X F_{X}-F}=\frac{X-\sqrt{X}}{X}, \\
c_{\mathrm{s}}^{2} & =\frac{\partial p / \partial X}{\partial \rho / \partial X}=\frac{F_{X}}{F_{X}+2 X F_{X X}}=1-\frac{1}{2 \sqrt{X}},
\end{aligned}
$$

where $F_{X} \equiv d F / d X$ and $F_{X X} \equiv d^{2} F / d X^{2}$. The sound speed comes from the equation describing the evolution of linear adiabatic perturbations in a k-essence dominated universe $[29,51]$ (a non-adiabatic perturbation of k-essence has been discussed in [52,53], here we only consider the case of adiabatic perturbation). From Equations (3) and (4), it has $c_{\mathrm{s}}^{2}=\frac{1+w_{\phi}}{2}$. Meanwhile, by considering the stability of solutions with respect to inhomogeneous perturbations as $1 \geq c_{\mathrm{s}}^{2} \geq 0$, it constrains the range $1 \geq w_{\phi} \geq-1$. It follows that the k-essence model in this paper does not permit phantom behaviour.

In the following discussion, we neglect baryonic matter $\rho_{b}$ and the radiation $\rho_{r}$ in the matter component. Subsequently, the Friedmann equations take the form

$$
\begin{array}{r}
H^{2}=\frac{1}{3}\left(\rho_{\mathrm{m}}+\rho_{\phi}\right), \\
\dot{H}=-\frac{1}{2}\left(\rho_{\mathrm{m}}+\rho_{\phi}+p_{\phi}\right),
\end{array}
$$

where $H=\dot{a} / a$ is the Hubble parameter, $\rho_{\phi}$ and $\rho_{m}$ are the DE and DM density, respectively. The equation of motion for the k-essence field is given by

$$
\left(F_{X}+2 X F_{X X}\right) \ddot{\phi}+3 H F_{X} \dot{\phi}+\left(2 X F_{X}-F\right) \frac{V_{\phi}}{V}=0,
$$

where $V_{\phi} \equiv d V / d \phi$. Equations (5) and (6) are usually transformed into an autonomous dynamical system when performing the phase-space and stability analysis. Being derived from the Friedmann eqs., we obtain $\frac{\ddot{a}}{a}=-\frac{1}{6}(3 p+\rho)$, which implies a continuous eq. $\dot{\rho}+3 H(p+\rho)=0$. Because, in this model, the density is composed by two parts, the dark energy density and the matter density, i.e., $\rho=\rho_{m}+\rho_{\phi}$, 
with the interaction $Q$ between $\mathrm{DM}$ and $\mathrm{DE}, \rho_{\phi}$ and $\rho_{m}$ do not separately satisfy conservation laws. Subsequently, the following two equations are conceived as:

$$
\begin{aligned}
\dot{\rho}_{\phi}+3 H\left(\rho_{\phi}+p_{\phi}\right) & =Q, \\
\dot{\rho}_{m}+3 H\left(\rho_{m}+p_{m}\right) & =-Q .
\end{aligned}
$$

Here, $p_{m}=0$. For $Q<0$, there is a transfer of energy from dark energy to dark matter. The case of $Q=0$, as in no interaction, was discussed in the former paper [38]. While, in this paper, the interaction is chosen by $Q=\alpha H \rho_{m}$, which means that the transformation between dark energy and dark matter happen, to some degree, in that circumstance. By Equations (8) and (9), to keep the physical dimensionality, we have to set $\alpha$ as a dimensionless parameter [33].

By setting the phase components, the auxiliary variables are defined as

$$
x=\dot{\phi}, \quad y=\frac{\sqrt{V(\phi)}}{\sqrt{3} H},
$$

in order to transform the cosmological Equations (5) and (6) into an autonomous dynamical system $\left\{x^{\prime}, y^{\prime}\right\}$, by considering (8) and (9), where the prime is the derivative with respect to $N=\ln a$. Subsequently, after solving the eqs. $\left\{x_{c}^{\prime}=0, y_{c}^{\prime}=0\right\}$, the critical points $\mathbf{X}_{c}=\left\{x_{c}, y_{c}\right\}$ are obtained. To discuss the stability of each critical point, we expand $\mathbf{X}=\{x, y\}$ around the critical points $\mathbf{X}_{\mathrm{c}}=\left\{x_{c}, y_{c}\right\}$ by setting $\{x, y\}^{T}=\left\{x_{c}, y_{c}\right\}^{T}+\mathbf{U}$ with the perturbational variables $\mathbf{U}$ (see, for example, Refs. [41,54-58]). Up to the first order we acquire $\mathbf{U}^{\prime}=\mathbf{M} \cdot \mathbf{U}$ with the $2 \times 2$ matrix $\mathbf{M}$ determined by

$$
\mathbf{M}=\left[\begin{array}{ll}
\frac{\partial x^{\prime}}{\partial x} & \frac{\partial x^{\prime}}{\partial y} \\
\frac{\partial y^{\prime}}{\partial x} & \frac{\partial y^{\prime}}{\partial y}
\end{array}\right] .
$$

The matrix $\mathbf{M}$ contains the coefficients of the perturbation equations, and thus its eigenvalues determine the stability of the critical points. In this 2-dim system, which has two eigenvalues of $M$, for hyperbolic critical points, all of the eigenvalues have real parts that are different from zero: sink for the negative real parts is stable, saddle for real parts of different sign is unstable, and source for positive real parts is unstable. However, for the convenience of calculation in this model, an alternative way to judge the stability of the critical points in a 2-dim system is given by the trace $\operatorname{tr} \mathbf{M}<0$ and determinant $\operatorname{det} \mathbf{M}>0$.

For the more general linear interaction form $Q=\alpha H \rho_{m}+\beta H \rho_{\phi}$, the autonomous dynamical equations are derived, as follows:

$$
\begin{aligned}
& x^{\prime}=\frac{\sqrt{3}}{2} \lambda x^{2} y-3 x+\frac{3 \sqrt{2}}{2}+\frac{\alpha}{x y^{2}}-\frac{1}{2} x \alpha+\frac{x \beta}{2}, \\
& y^{\prime}=\frac{1}{4} y\left(-2 \sqrt{3} \lambda x y+6-3 \sqrt{2} x y^{2}+3 x^{2} y^{2}\right),
\end{aligned}
$$

for $x>0$; while, for $x<0$, the equations turn out to be

$$
\begin{aligned}
& x^{\prime}=\frac{\sqrt{3}}{2} \lambda x^{2} y-3 x-\frac{3 \sqrt{2}}{2}+\frac{\alpha}{x y^{2}}-\frac{1}{2} x \alpha+\frac{x \beta}{2}, \\
& y^{\prime}=\frac{1}{4} y\left(-2 \sqrt{3} \lambda x y+6+3 \sqrt{2} x y^{2}+3 x^{2} y^{2}\right),
\end{aligned}
$$

where $\lambda \equiv-V_{\phi} / V^{\frac{3}{2}}=$ const, by assuming $V(\phi)=4 \lambda^{-2} \phi^{-2}$ in this paper. Because it is hard to obtain an analytic solution as a critical point from the equations above with three parameters $\lambda, \alpha$, and $\beta$, it has to be simplified by two cases in the next step, i.e., $Q_{1}=\alpha H \rho_{m}$ and $Q_{2}=\beta H \rho_{\phi}$, respectively. 
However, we only analyze $Q_{1}$ in this paper due to the unsatisfactory for $Q_{2}$. Besides that, the values of $\alpha$ and $\beta$ are constrained in certain ranges, which are imposed for the observations [18-20].

\section{The Analysis of Stability for This Dark Energy Model with Interaction $Q=\alpha H \rho_{m}$}

In this case of $Q=\alpha H \rho_{m}$, when $x>0$, Equations (5) and (6) are simplified, as follows:

$$
\begin{aligned}
& x^{\prime}=\frac{\sqrt{3}}{2} \lambda x^{2} y-3 x+\frac{3 \sqrt{2}}{2}+\frac{\alpha}{x y^{2}}-\frac{1}{2} x \alpha, \\
& y^{\prime}=\frac{1}{4} y\left(-2 \sqrt{3} \lambda x y+6-3 \sqrt{2} x y^{2}+3 x^{2} y^{2}\right),
\end{aligned}
$$

By (16) and (17), the corresponding critical points $\left\{x_{c}, y_{c}\right\}$ are

$$
\begin{aligned}
& P_{1}=\left\{x_{c}, y_{c}\right\}=\left\{\frac{\sqrt{3}}{-\lambda+\sqrt{6}}, \frac{\sqrt{6}}{3}(-\lambda+\sqrt{6})\right\}, \\
& P_{2}=\left\{x_{c}, y_{c}\right\}=\left\{\frac{\sqrt{3}}{\lambda+\sqrt{6}},-\frac{\sqrt{6}}{3}(\lambda+\sqrt{6})\right\}, \\
& P_{3}=\left\{x_{c}, y_{c}\right\}=\left\{\frac{\sqrt{2}\left(9+6 \alpha+\alpha^{2}\right)}{-2 \lambda^{2} \alpha+9+\alpha^{2}+6 \alpha}, \frac{\sqrt{6}}{6 \lambda(\alpha+3)}\left(-2 \lambda^{2} \alpha+9+\alpha^{2}+6 \alpha\right)\right\} .
\end{aligned}
$$

While, when $x<0$, we have

$$
\begin{aligned}
& x^{\prime}=\frac{\sqrt{3}}{2} \lambda x^{2} y-3 x-\frac{3 \sqrt{2}}{2}+\frac{\alpha}{x y^{2}}-\frac{1}{2} x \alpha, \\
& y^{\prime}=\frac{1}{4} y\left(-2 \sqrt{3} \lambda x y+6+3 \sqrt{2} x y^{2}+3 x^{2} y^{2}\right),
\end{aligned}
$$

Additionally, there are other three solutions as the critical points, as follows:

$$
\begin{aligned}
& P_{4}=\left\{x_{c}, y_{c}\right\}=\left\{\frac{-\sqrt{3}}{\lambda+\sqrt{6}}, \frac{\sqrt{6}}{3}(\lambda+\sqrt{6})\right\}, \\
& P_{5}=\left\{x_{c}, y_{c}\right\}=\left\{\frac{-\sqrt{3}}{-\lambda+\sqrt{6}},-\frac{\sqrt{6}}{3}(-\lambda+\sqrt{6})\right\}, \\
& P_{6}=\left\{x_{c}, y_{c}\right\}=\left\{\frac{-\sqrt{2}\left(9+6 \alpha+\alpha^{2}\right)}{-2 \lambda^{2} \alpha+9+\alpha^{2}+6 \alpha}, \frac{-\sqrt{6}}{6 \lambda(\alpha+3)}\left(-2 \lambda^{2} \alpha+9+\alpha^{2}+6 \alpha\right)\right\} .
\end{aligned}
$$

The corresponding density parameter, the EoS, the sound speed, and the deceleration parameter are reexpressed as, respectively,

$$
\begin{aligned}
\Omega_{\phi} & =\frac{1}{2} x^{2} y^{2} \\
w_{\phi} & =1-\sqrt{2}|x|^{-1}, \\
c_{\mathrm{s}}^{2} & =1-\frac{\sqrt{2}}{2}|x|^{-1}, \\
q & =\frac{1}{2}+\frac{3}{4} x^{2} y^{2}-\frac{3 \sqrt{2}}{4}|x| y^{2} .
\end{aligned}
$$

Equations (16), (17), (21) and (22) form the self-autonomous dynamical systems, which are valid in the whole phase-space, not only at the critical points. The critical points $\left\{x_{c}, y_{c}\right\}$ of the autonomous system are obtained by setting the left-hand sides of the equations to zero, namely by solving $\mathbf{X}^{\prime}=\left(x^{\prime}, y^{\prime}\right)^{T}=0$. Six critical points are obtained, as shown in Table 1 , in which we also present the necessary conditions for their existences and stabilities, as well as the corresponding 
cosmological quantities, $c_{\mathrm{S}}^{2}, \Omega_{\phi}, w_{\phi}$, and $q$. With these cosmological quantities, we can investigate the final state of the universe and discuss whether there exists acceleration expansion or not. Physically, it requires $0 \leq \Omega_{\phi} \leq 1$, so the auxiliary variables $x$ and $y$ are constrained as $0 \leq \frac{1}{2} x^{2} y^{2} \leq 1$. In order to comply with the accelerated expansion, it requires $w_{\phi}<-\frac{1}{3}$. When considering the sound speed, it has to be $0 \leq c_{\mathrm{s}}^{2} \leq 1$. For the existence, it means $y>0$, and $x<0$ or $x>0$ for each case. The stability means $\operatorname{det} M>0$ and $\operatorname{tr} M<0$, instead of the analysis by each eigenvalue.

For the power law k-essence dark energy presented in this paper, the specific expression of the $\mathbf{M}$, $\operatorname{det} \mathbf{M}$, and $\operatorname{tr} \mathbf{M}$ are as follows:

$$
\begin{aligned}
& \mathbf{M}=\left[\begin{array}{cc}
-\frac{\alpha}{x^{2} y^{2}}-\frac{1}{2} \alpha+\sqrt{3} \lambda x y-3 & -\frac{2 \alpha}{y^{3} x}+\frac{\sqrt{3}}{2} \lambda x^{2} \\
\frac{1}{4} y\left(-2 \sqrt{3} \lambda y-3 \sqrt{2} y^{2}+6 x y^{2}\right) & -\sqrt{3} \lambda x y+\frac{3}{2}-\frac{9 \sqrt{2}}{4} x y^{2}+\frac{9}{4} x^{2} y^{2}
\end{array}\right], \\
& \operatorname{det} M=-\frac{9}{4} \lambda^{2} x^{2} y^{2}+\frac{9 \sqrt{3}}{2} \lambda x y-\frac{15 \sqrt{6}}{8} \lambda x^{2} y^{3}+\frac{3 \sqrt{3}}{2} \lambda x^{3} y^{3}-\frac{9}{2}+\frac{27 \sqrt{2}}{4} x y^{2} \\
&-\frac{27}{4} x^{2} y^{2}+\frac{\sqrt{3}}{2} x y \alpha \lambda+\frac{9 \sqrt{2}}{8} x y^{2} \alpha-\frac{9}{8} x^{2} y^{2} \alpha-\frac{3 \alpha}{2 x^{2} y^{2}}+\frac{3 \sqrt{2} \alpha}{4 x}, \\
& \operatorname{tr} M= \frac{-\alpha}{x^{2} y^{2}}-\frac{\alpha}{2}-\frac{3}{2}-\frac{9 \sqrt{2}}{4} x y^{2}+\frac{9}{4} x^{2} y^{2},
\end{aligned}
$$

for the case of $x>0$; and,

$$
\begin{aligned}
\mathbf{M}= & {\left[\begin{array}{cc}
-\frac{\alpha}{x^{2} y^{2}}-\frac{\alpha}{2}+\sqrt{3} \lambda x y-3 & \frac{-2 \alpha}{y^{3} x}+\frac{\sqrt{3}}{2} \lambda x^{2} \\
\frac{1}{4} y\left(-2 \sqrt{3} \lambda y+3 \sqrt{2} y^{2}+6 x y^{2}\right) & -\sqrt{3} \lambda x y+\frac{3}{2}+\frac{9 \sqrt{2}}{4} x y^{2}+\frac{9}{4} x^{2} y^{2}
\end{array}\right], } \\
\operatorname{det} M= & -\frac{9}{4} \lambda^{2} x^{2} y^{2}+\frac{9 \sqrt{3}}{2} \lambda x y+\frac{15 \sqrt{6}}{8} \lambda x^{2} y^{3}+\frac{3 \sqrt{3}}{2} \lambda x^{3} y^{3}-\frac{9}{2}-\frac{27 \sqrt{2}}{4} x y^{2}-\frac{27}{4} x^{2} y^{2} \\
& +\frac{\sqrt{3}}{2} x y \alpha \lambda-\frac{9 \sqrt{2}}{8} x y^{2} \alpha-\frac{9}{8} x^{2} y^{2} \alpha-\frac{3 \alpha}{2 x^{2} y^{2}}-\frac{3 \sqrt{2} \alpha}{4 x} \\
\operatorname{tr} M= & \frac{-\alpha}{x^{2} y^{2}}-\frac{\alpha}{2}-\frac{3}{2}+\frac{9 \sqrt{2}}{4} x y^{2}+\frac{9}{4} x^{2} y^{2},
\end{aligned}
$$

for the case of $x<0$.

According to the stability conditions of critical points by the determinant and trace, together with those cosmological quantities, we obtain the value range of $\lambda$ and $\alpha$ in the parameter plane, which makes the critical points stable and causes accelerated expansion, as shown in Table 1. At first, $P_{2}$ and $P_{5}$ are excluded by using existence condition. Based on the range of parameters presented in Figure 1a, we plot the stable point $P_{1}$ and its evolutionary trajectory for $\lambda=0.5$ and $\alpha=0.01$ in Figure $1 \mathrm{~b}$, as well as $P_{3}, P_{4}$, and $P_{6}$ (see Figures 2-4) with some certain pairs of parameters $\lambda$ and $\alpha$, respectively, in order to have a visual understanding of the evolutionary behavior near critical points. Especially, $P_{3}$ and $P_{6}$ are spiral attractors, which have spiral evolutionary trajectories around them. Additionally, the evolutionary trajectories of the cosmological quantities are shown in Figures 5 and 6 . Below, we will analyze these stable points $P_{1}, P_{3}, P_{4}$, and $P_{6}$ one-by-one.

For $P_{1}$, it has $\Omega_{\phi}=1$; the universe will be dominated by k-essence dark energy. If $\lambda=0$, then the k-essence would behave like a cosmological constant. The deceleration parameter $q=-1+\frac{\sqrt{6}}{2} \lambda$. The final state of the universe depends on the potential parameter $\lambda$, i.e., the universe expansion would speed up if $\lambda<\frac{\sqrt{6}}{3}$, it would expand at constant-speed if $\lambda=\frac{\sqrt{6}}{3}$, and the universe expansion would slow down if $\frac{\sqrt{6}}{3}<\lambda$. 
For $P_{3}, \Omega_{\phi}=\frac{(3+\alpha)^{2}}{6 \lambda^{2}}$, the universe will be dominated by both k-essence dark energy and dark matter. When $\alpha$ and $\lambda$ satisfy $\frac{2 \alpha \lambda^{2}}{(3+\alpha)^{2}}=-1$, lying on the top edge of the grey region in Figure 2a, the k-essence will behave like a cosmological constant. The evolutionary trajectory in the phase space shown in Figure $2 \mathrm{~b}$ will be spiral around $P_{3}$, finally converging to the attractor $P_{3}$. The deceleration parameter $q=\frac{1}{2}+\frac{\alpha}{2}$. The final state of the universe depends on the coupling parameter $\alpha$, i.e., the expansion of the universe will speed up if $-3<\alpha<-1$, will expand in a constant speed if $\alpha=-1$, and will slow down if $-1<\alpha<0$, respectively.

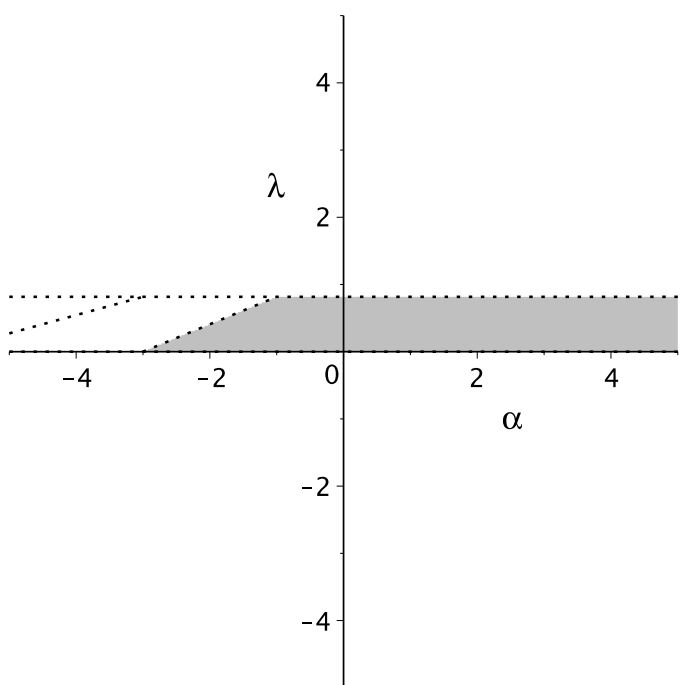

(a)

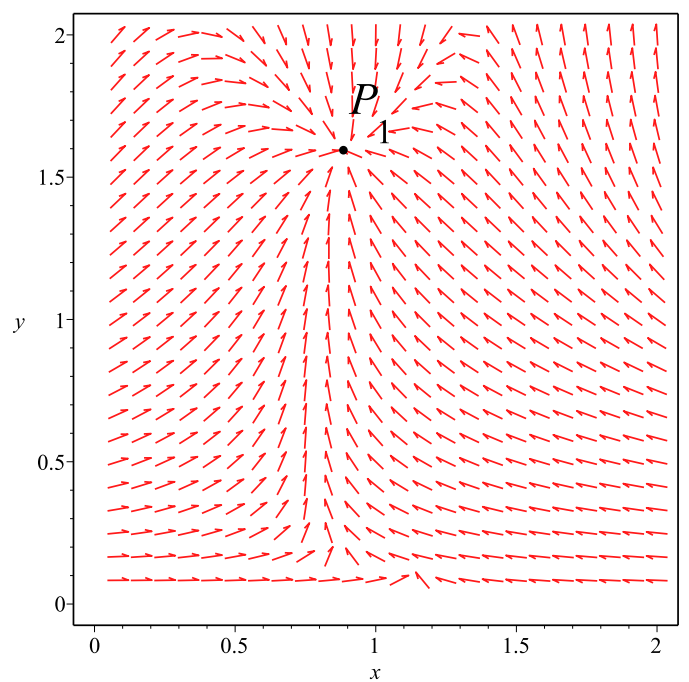

(b)

Figure 1. (a) The value ranges for parameters $\lambda$ and $\alpha$ to make the critical point $P_{1}$ exist and stable, which is also constrained by other cosmological quantities, when $x>0$. (b) The phase plane for $\lambda=0.5$ and $\alpha=0.01$ around the attractor $P_{1}=(0.888,1.592)$ when $x>0$.

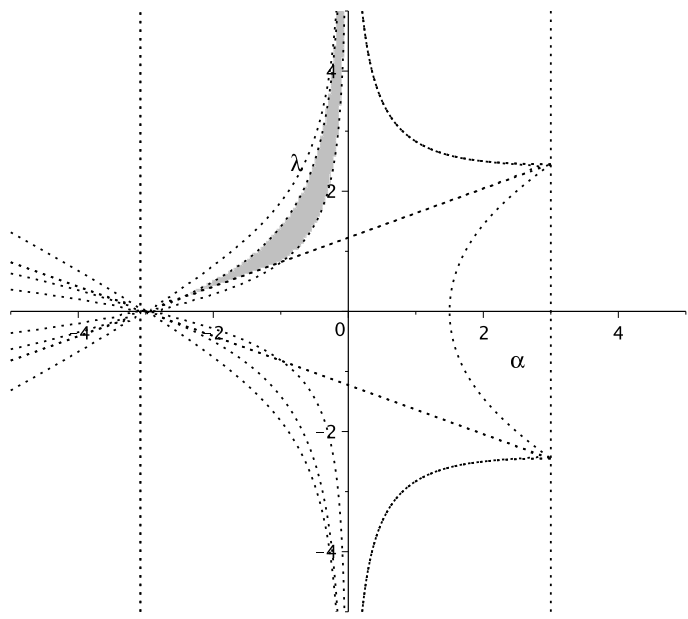

(a)

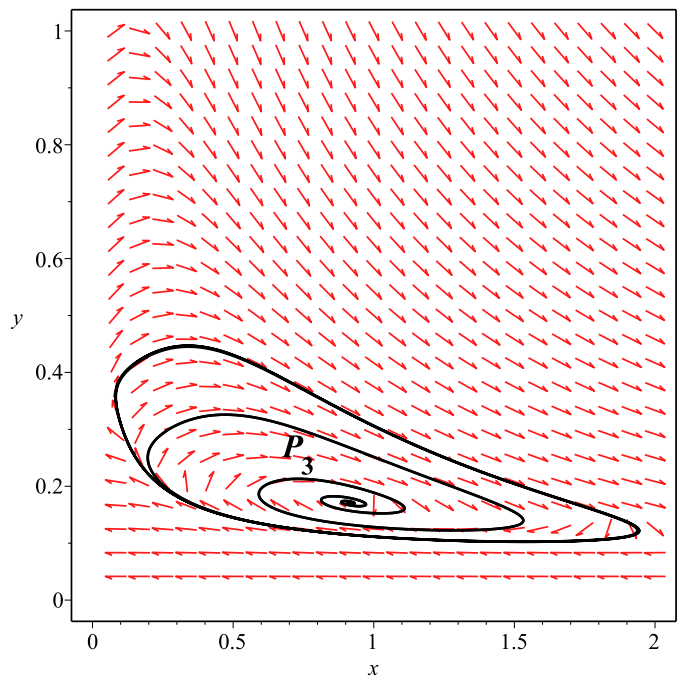

(b)

Figure 2. (a) The value range for parameters $\lambda$ and $\alpha$ to make the critical point $P_{3}$ exist and stable, also constrained by other cosmological quantities, when $x>0$. (b) The phase plane for $\lambda=11$ and $\alpha=-0.02$ around the attractor $P_{3}=(0.915,0.171)$ when $x>0$. 
For $x<0$, the results are quite like the three critical points that are investigated above. Among the three critical points, $P_{4}$ and $P_{6}$ are attractors for some $\lambda$ and $\alpha$, as displayed in Figures 3 and 4 . Those two points are also physically meaningful; while, $P_{5}$ does not.

For $P_{4}$, it has $\Omega_{\phi}=1$, the universe will be dominated by k-essence dark energy. If $\lambda=0$, the k-essence will behave like a cosmological constant. The deceleration parameter $q=-1-\frac{\sqrt{6}}{2} \lambda$, which indicates that the final state of the universe depends on the potential, i.e., the universe expansion will speed up if $\lambda>-\frac{\sqrt{6}}{3}$, will expand with constant-speed if $\lambda=-\frac{\sqrt{6}}{3}$, and it will slow down if $-\frac{\sqrt{6}}{3}>\lambda$.

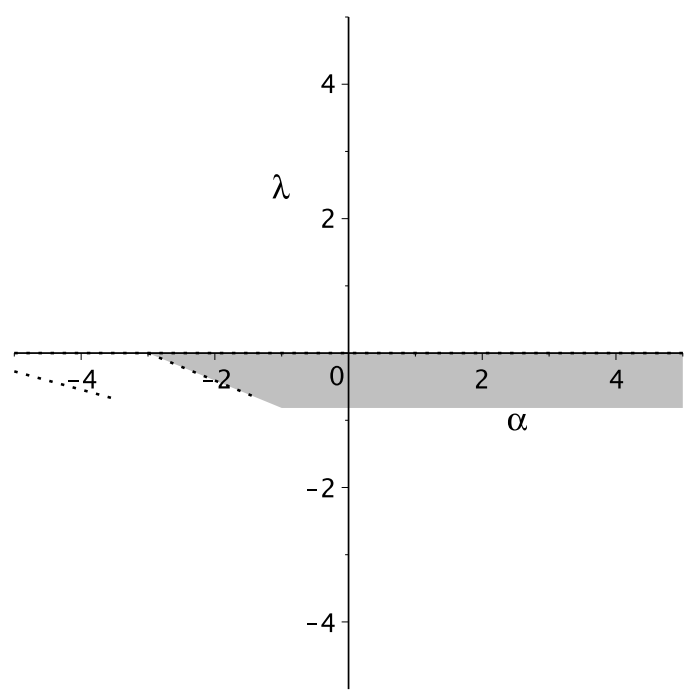

(a)

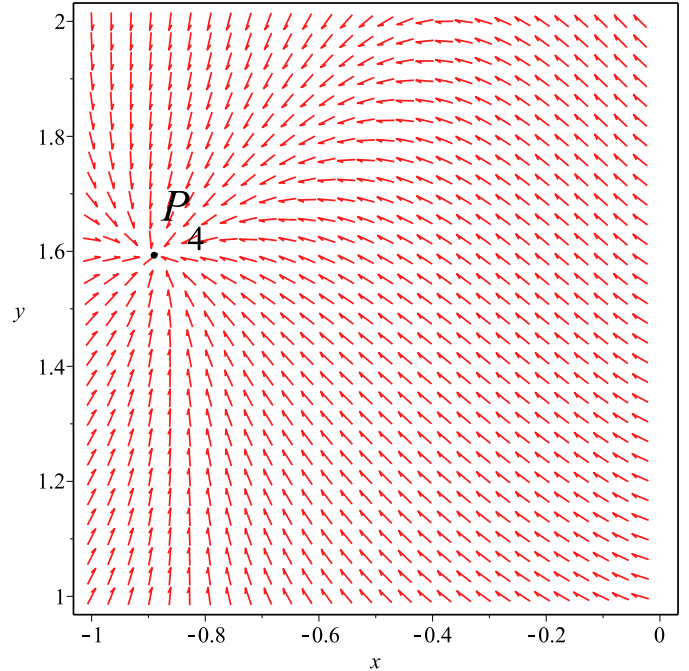

(b)

Figure 3. (a) The value range for parameters $\lambda$ and $\alpha$ to make the critical point $P_{4}$ exist and stable, also being constrained by other cosmological quantities, when $x<0$. (b) The phase plane for $\lambda=-0.5$ and $\alpha=0.01$ around the attractor $P_{4}=(-0.888,1.592)$ when $x<0$.

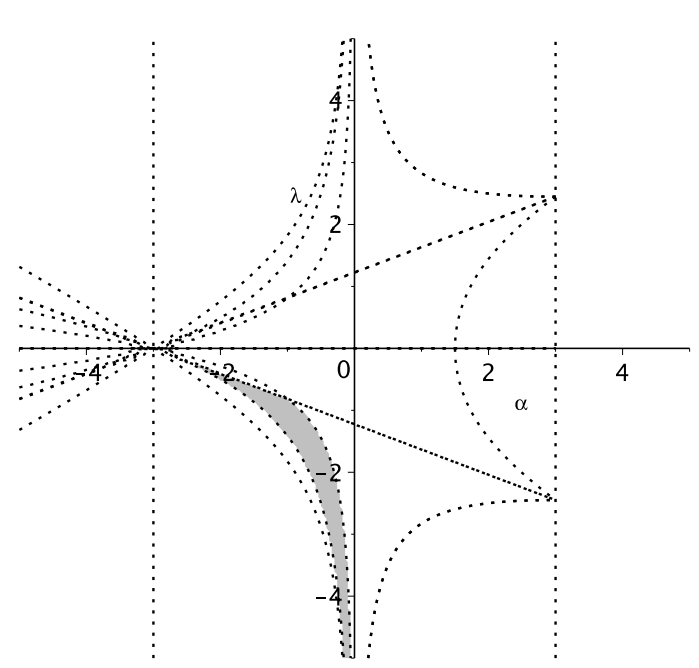

(a)

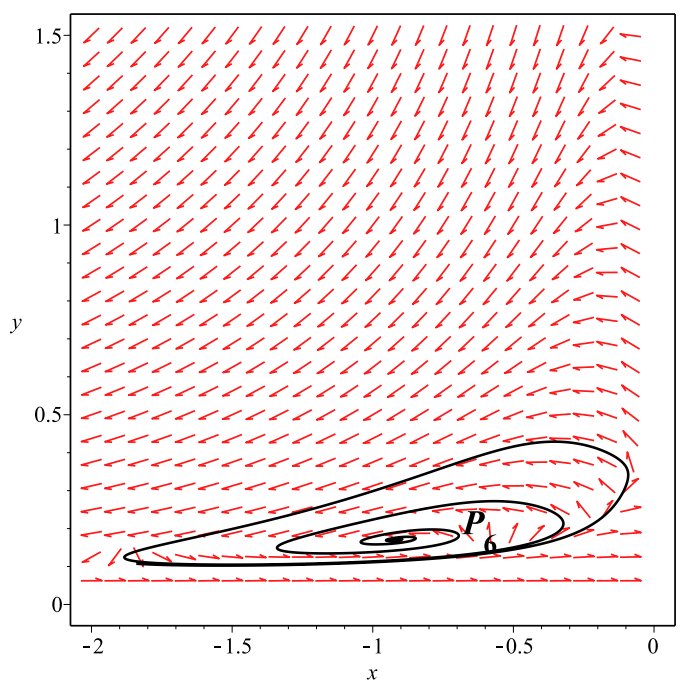

(b)

Figure 4. (a) The value range for parameters $\lambda$ and $\alpha$ to make the critical point $P_{6}$ exist and stable, also constrained by other cosmological quantities, when $x<0$. (b) The phase plane for $\lambda=-11$ and $\alpha=-0.02$ around the attractor $P_{6}=(-0.915,0.171)$ when $x<0$. 
For $P_{6}, \Omega_{\phi}=\frac{(3+\alpha)^{2}}{6 \lambda^{2}}$, the universe will be dominated by both k-essence and dark matter. When $\alpha$ and $\lambda$ satisfy $\frac{2 \alpha \lambda^{2}}{(3+\alpha)^{2}}=-1$, the k-essence will behave like a cosmological constant. In the phase space, the evolutionary trajectory will be spiral around $P_{6}$, and then finally converge to the attractor point. The deceleration parameter $q=\frac{1}{2}+\frac{\alpha}{2}$, which indicates that the final state of the universe depends on the dark matter: the universe will speed up if $\alpha<-1$, will expand with constant-speed if $\alpha=-1$, and it will slow down if $\alpha>-1$.

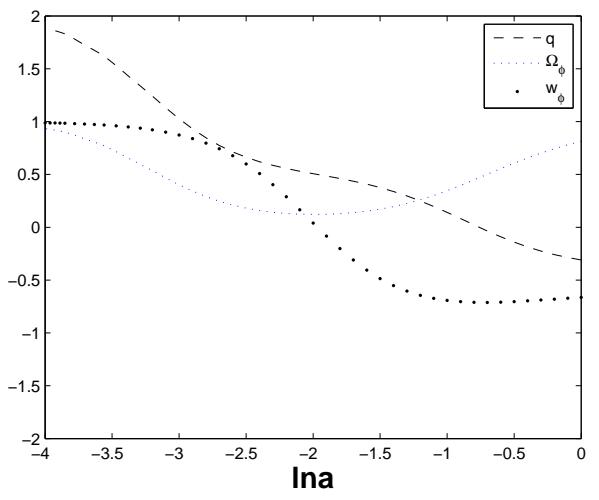

(a)

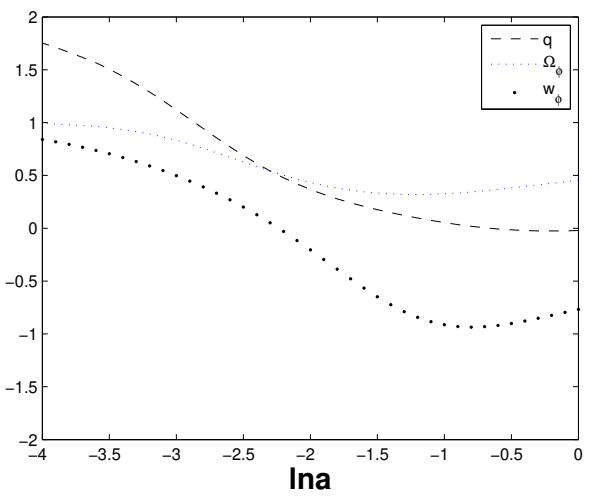

(b)

Figure 5. (a) The evolutions of $\Omega_{\phi}, w_{\phi}$ and the deceleration parameter $q$ by the initial condition $\left(x_{0}, y_{0}\right)=(0.85,1.5)$ around $P_{1}$ corresponding to $(\lambda, \alpha)=(0.5,0.01)$. (b) The evolutions of $\Omega_{\phi}, w_{\phi}$ and the deceleration parameter $q$ by the initial condition $\left(x_{0}, y_{0}\right)=(0.9,1.0)$ around $P_{3}$ corresponding to $(\lambda, \alpha)=(1.2,-0.8)$.

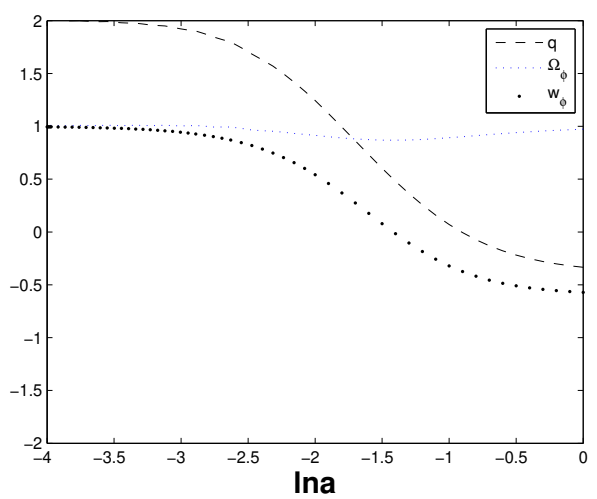

(a)

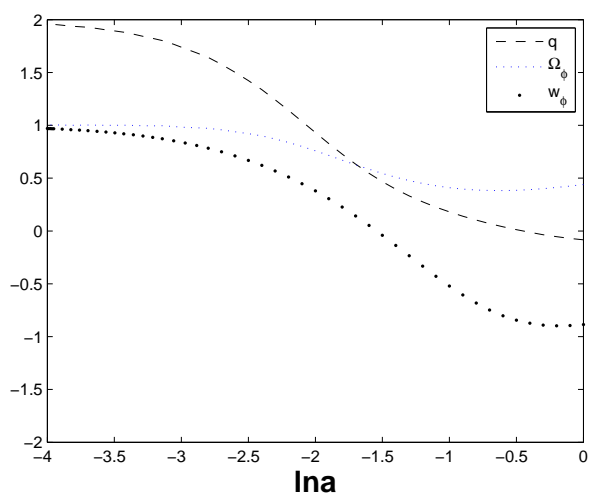

(b)

Figure 6. (a) The evolutions of $\Omega_{\phi}, w_{\phi}$ and the deceleration parameter $q$ by the initial condition $\left(x_{0}, y_{0}\right)=(-0.9,1.55)$ around $P_{4}$ corresponding to $(\lambda, \alpha)=(-0.5,0.01)$. (b) The evolutions of $\Omega_{\phi}, w_{\phi}$ and the deceleration parameter $q$ by the initial condition $\left(x_{0}, y_{0}\right)=(-0.75,1.25)$ around $P_{6}$ corresponding to $(\lambda, \alpha)=(-1,-0.9)$.

From Figures 5 and 6, it is not difficult to see that the density parameter of DE $\Omega_{\phi}$ continuously varies, which indicates that there is the exchange of energy between dark energy and dark matter by the interaction $\mathrm{Q}$, and, at present, the universe is composed by both DE and DM. Meanwhile, the deceleration parameter $q$ evolves from positive to negative values, which shows that the universe experiences a decelerating expansion in the past, and then transforms to an accelerating expansion at present, and keeps on speeding up into the future. Especially, in Figures $5 \mathrm{~b}$ and $6 \mathrm{~b}$, the $w_{\phi}>0$ happens in the early time, which means that, in early time, the dark energy performs in a relativistic matter, which provides positive pressure, and acts as the attraction force to enhance the structure 
formation. All four evolutional figures show the $w_{\phi}<-1 / 3$ in the late time universe, which indicates that this k-essence DE model could explain the accelerating expansion of the universe.

Table 1. The existence and stability conditions for six critical points, and the cosmological quantities in form of the parameters $\lambda$ and $\alpha$ in each critical point.

\begin{tabular}{|c|c|c|c|c|c|c|}
\hline Name & Existence & Stability & $\mathbf{0} \leq c_{s}^{2} \leq \mathbf{1}$ & $\mathbf{0} \leq \mathbf{\Omega}_{\phi} \leq \mathbf{1}$ & $\omega_{\phi}<-\frac{1}{3}$ & $q$ \\
\hline$P_{1}$ & $\lambda<\sqrt{6}$ & $\begin{array}{l}\sqrt{6} \lambda-3-\alpha<0 \text { and } \\
3 \sqrt{6} \lambda-2 \alpha-12<0\end{array}$ & $\frac{\lambda}{\sqrt{6}}$ & 1 & $\frac{\sqrt{6} \lambda-3}{3}$ & $\frac{\sqrt{6}}{2} \lambda-1$ \\
\hline$P_{2}$ & none & $\begin{array}{l}\sqrt{6} \lambda+3+\alpha<0 \text { and } \\
3 \sqrt{6} \lambda+2 \alpha+12>0\end{array}$ & $-\frac{\lambda}{\sqrt{6}}$ & 1 & $\frac{\sqrt{6} \lambda+3}{-3}$ & $-1-\frac{\sqrt{6}}{2} \lambda$ \\
\hline$P_{3}$ & $\begin{array}{l}(3+\alpha)^{2}>2 \lambda^{2} \alpha \\
\text { and } \lambda(\alpha+3)>0\end{array}$ & $\begin{array}{c}\lambda\left((3+\alpha)^{2}-6 \lambda^{2}\right)<0 \text { and } \\
-6 \lambda^{2} \alpha+9 \alpha^{2}+2 \alpha^{3}<27\end{array}$ & $\frac{\alpha \lambda^{2}}{(3+\alpha)^{2}}+\frac{1}{2}$ & $\frac{(3+\alpha)^{2}}{6 \lambda^{2}}$ & $\frac{2 \alpha \lambda^{2}}{(3+\alpha)^{2}}$ & $\frac{1}{2}+\frac{\alpha}{2}$ \\
\hline$P_{4}$ & $\lambda>-\sqrt{6}$ & $\begin{array}{l}\sqrt{6} \lambda+3+\alpha>0 \text { and } \\
3 \sqrt{6} \lambda+2 \alpha+12>0\end{array}$ & $-\frac{\lambda}{\sqrt{6}}$ & 1 & $\frac{\sqrt{6} \lambda+3}{-3}$ & $-1-\frac{\sqrt{6}}{2} \lambda$ \\
\hline$P_{5}$ & none & $\begin{array}{c}\left(\lambda-\frac{3}{\sqrt{6}}-\frac{\alpha}{\sqrt{6}}\right)\left(\frac{\sqrt{6}-\lambda}{\sqrt{6}}\right)<0 \\
\text { and } 3 \sqrt{6} \lambda-2 \alpha-12<0\end{array}$ & $\frac{\sqrt{6}}{6} \lambda$ & 1 & $\frac{\sqrt{6} \lambda-3}{3}$ & $\frac{\sqrt{6}}{2} \lambda-1$ \\
\hline$P_{6}$ & $\begin{array}{c}(3+\alpha)^{2}>2 \lambda^{2} \alpha \\
\text { and } \lambda(\alpha+3)<0\end{array}$ & $\begin{array}{c}\lambda\left((3+\alpha)^{2}-6 \lambda^{2}\right)>0 \text { and } \\
-6 \lambda^{2} \alpha+9 \alpha^{2}+2 \alpha^{3}<27\end{array}$ & $\frac{\alpha \lambda^{2}}{(3+\alpha)^{2}}+\frac{1}{2}$ & $\frac{(3+\alpha)^{2}}{6 \lambda^{2}}$ & $\frac{2 \alpha \lambda^{2}}{(3+\alpha)^{2}}$ & $\frac{1}{2}+\frac{\alpha}{2}$ \\
\hline
\end{tabular}

\section{The Geometric Diagnostic of Statefinder Hierarchy}

Because the $\Lambda \mathrm{CDM}$ model is the best fitting for observations until now, the statefinder pair is a way for distinguishing a certain model from the $\Lambda$ CDM model, by showing the "distance" of trajectories in the $s-r$ plane from the spatially flat $\Lambda \mathrm{CDM}$ model scenario which is a fixed point $\left.\{s, r\}\right|_{\Lambda C D M}=\{0,1\}$. Beyond the Hubble parameter $H=\frac{\dot{a}}{a}$ and the deceleration parameter $q=-\frac{a \ddot{a}}{\dot{a}^{2}}$, the third order derivative $r=\frac{\dddot{a}}{a H^{3}}$, together with a combination of $r$ and $q$, which is $s=\frac{r-1}{3\left(q-\frac{1}{2}\right)}$, become the cosmological diagnostic pair $\{r, s\}$. In terms of $\Omega_{\phi}$ and $w$, the statefinder pair has the following form:

$$
\begin{aligned}
r & =1+\frac{9}{2} \Omega_{\phi} w(1+w)-\frac{3}{2} \Omega_{\phi} \frac{\dot{w}}{H^{\prime}} \\
s & =1+w-\frac{1}{3} \frac{\dot{w}}{w H} .
\end{aligned}
$$

Further, the statefinder hierarchy is an extension of the statefinder pair, which comes from the view point of higher derivatives of the expansion factor $d^{n} a / d t^{n}$, in Taylor expanded: $(1+z)^{-1}=$ $\frac{a(t)}{a_{0}}=1+\sum \frac{A_{n}\left(t_{0}\right)}{n !}\left[H_{0}\left(t-t_{0}\right)\right]^{n}$, where $A_{n}=\frac{a(t)^{(n)}}{a(t) H^{n}}, n \in \mathbb{N}$, and $a(t)^{(n)}=d^{n} a(t) / d t^{n}$. By using $A_{n}$, the series $S_{n}$ and $S_{n}^{(1)}$ are constructed, as follows. In a spatially flat universe with pressureless matter and a cosmological constant, such as $\Lambda C D M$ model, $A_{n}$ could be expressed by parameter $q$ or $\Omega_{m}$, where $\Omega_{m}=\frac{2}{3}(1+q)$, as follows:

$$
\begin{aligned}
& A_{2}=1-\frac{3}{2} \Omega_{m}, \\
& A_{3}=1, \\
& A_{4}=1-\frac{3^{2}}{2} \Omega_{m}, \\
& A_{5}=1+3 \Omega_{m}+\frac{3^{3}}{2} \Omega_{m}^{2} .
\end{aligned}
$$


Then the statefinder hierarchy $S_{n}$ is defined as

$$
\begin{aligned}
& S_{2}=A_{2}+\frac{3}{2} \Omega_{m}, \\
& S_{3}=A_{3} \\
& S_{4}=A_{4}+\frac{3^{2}}{2} \Omega_{m} \\
& S_{5}=A_{5}-3 \Omega_{m}-\frac{3^{3}}{2} \Omega_{m}^{2} .
\end{aligned}
$$

Finally, it derived the null diagnostic for the $\Lambda C D M$ model, the $S_{n}^{(1)}$, as follows:

$$
\begin{aligned}
& S_{3}^{(1)}=S_{3}, \\
& S_{4}^{(1)}=A_{4}+3(1+q), \\
& S_{5}^{(1)}=A_{5}-2(4+3 q)(1+q) .
\end{aligned}
$$

For the $\Lambda C D M$ model, it always has $\left.S_{n}\right|_{\Lambda C D M}=1$ and $\left.S_{n}^{(1)}\right|_{\Lambda C D M}=1$. In this paper, we focus on how parameters $\lambda$ and $\alpha$ effect the statefinder hierarchy $S_{3}^{(1)}$ and $S_{4}^{(1)}$ for the k-essence model with coupling $Q=\alpha H \rho_{m}$. We can obtain the following expressions with interaction $Q$ :

$$
\begin{aligned}
S_{3}^{(1)}= & 1+\frac{9}{2} \Omega_{\phi} w_{\phi}\left(1+w_{\phi}\right)-\frac{3}{2} \Omega_{\phi} w_{\phi}^{\prime}-\frac{3 w_{\phi} Q}{2 H \rho} \\
S_{4}^{(1)}= & 1-\frac{9}{4} w_{\phi} \Omega_{\phi}^{2}\left[3 w_{\phi}\left(1+w_{\phi}\right)-w_{\phi}^{\prime}\right]-\frac{3}{4} \Omega_{\phi}\left[w_{\phi}\left(21+39 w_{\phi}+18 w_{\phi}^{2}\right)-\left(13+18 w_{\phi}\right) w_{\phi}^{\prime}\right. \\
& \left.+2 w_{\phi}^{\prime \prime}\right]+\frac{3 w_{\phi} Q}{2 H \rho}\left(2+3 w_{\phi}\right)-\frac{3 w_{\phi} Q^{\prime}}{2 H \rho}-\frac{3 w_{\phi}^{\prime} Q}{H \rho} .
\end{aligned}
$$

The former methods for analyzing the statefinder pair $\{r, s\}$ or the statefinder hierarchy are mainly around purely kinetic k-essence dark energy models, by finding the relations among $F_{X}, w_{\phi}$ and $a(t)$. For the potential, in this paper, it is not as a constant as in PKK and the analytic relation cannot be derived directly; instead, the statefinder hierarchy should be represented by phase components $\{x, y\}$. Along with the numerical method presented in [49], after substituting (16) and (17) into (49) and (50) for $x>0$, and (21) and (22) into (49) and (50) for $x<0$, respectively, the statefinder hierarchy $S_{3}^{(1)}$ and $S_{4}^{(1)}$ could be expressed by variables $x, y$ with parameters $\lambda, \alpha$. We choose the initial points $\left\{x_{0}, y_{0}\right\}$ around the attractors separately, and then adjust $\lambda$ with a constant $\alpha$, whereas adjust $\alpha$ with a constant $\lambda$, in order to see the effect on statefinder hierarchy for each by the figures.

For $P_{1}$, from Figure 7 , by changing the value of potential parameter $\lambda$ with a fixed coupling parameter $\alpha=0.01$, the difference of both $S_{3}^{(1)}$ and $S_{4}^{(1)}$ evolutionary curves are obvious. However, in Figure 8, there is nearly no difference in both $S_{3}^{(1)}$ and $S_{4}^{(1)}$ cases, with the same value of $\lambda=0.5$, but different values of $\alpha$. It means that the statefinder hierarchy $S_{3}^{(1)}$ and $S_{4}^{(1)}$ are more sensible to the potential parameter $\lambda$ than $\alpha$ around $P_{1}$.

Oppositely, for $P_{3}$, there is no difference by changing the potential parameter $\lambda$ under the same value $\alpha=-0.02$ for both $S_{3}^{(1)}$ and $S_{4}^{(1)}$ in Figure 9 , while the statefinder hierarchy shows the sensibility to the coupling parameter $\alpha$ under the same value of $\lambda=11$ in Figure 10. That is to say, curves with same value of $\alpha$ perform alike, while $\lambda$ makes little effect around $P_{3}$.

For the case of $x<0$, i.e., $P_{4}$ and $P_{6}$, the evolutionary curves of the statefinder hierarchy $S_{3}^{(1)}$ and $S_{4}^{(1)}$ are analogous to the $P_{1}$ and $P_{3}$, respectively. For all of cases above, the statefinder hierarchy $S_{3}^{(1)}$ and $S_{4}^{(1)}$ of the k-essence DE model in this paper can tell the difference from the $\Lambda$ CDM model, which is a straight line in the figures. 


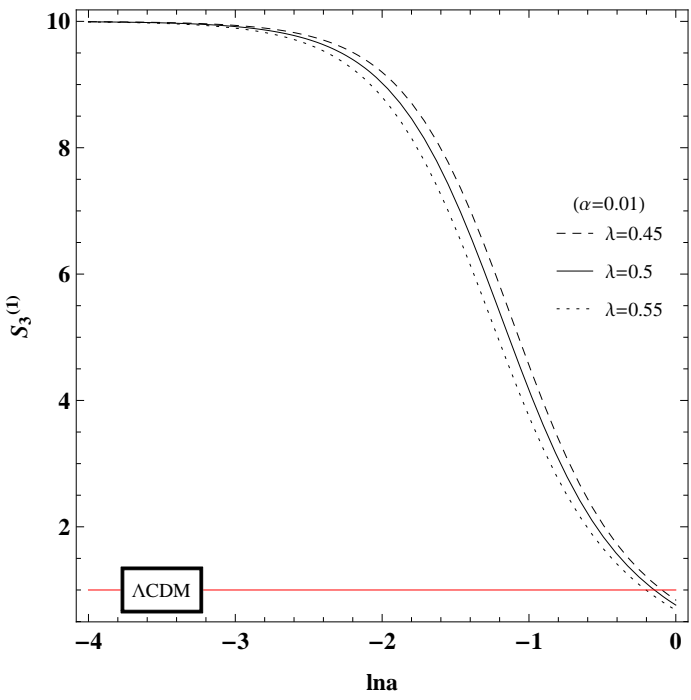

(a)

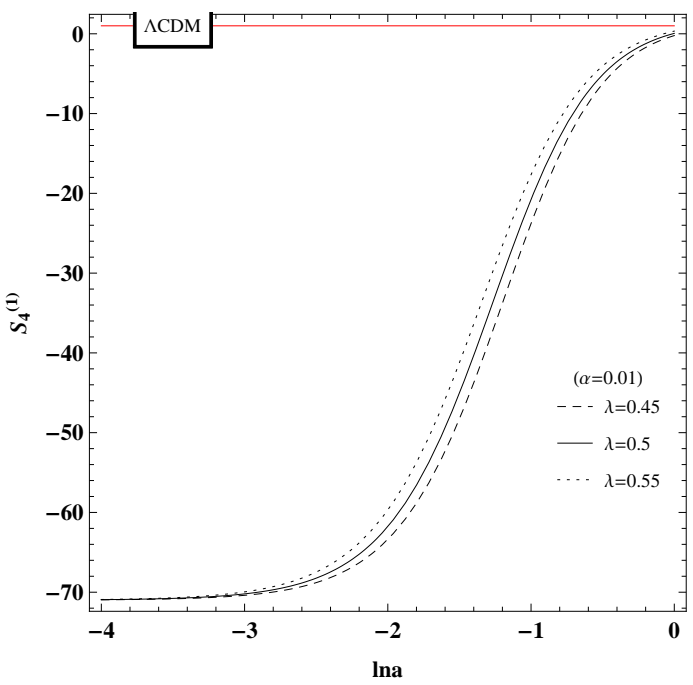

(b)

Figure 7. (a) Graph of $S_{3}^{(1)}$ for $P_{1}$ with different parameters $\lambda$ and fixed $\alpha=0.01$. (b) Graph of $S_{4}^{(1)}$ for $P_{1}$ with different parameters $\lambda$ and fixed $\alpha=0.01$. The initial point is $x_{0}=1, y_{0}=1.2$.

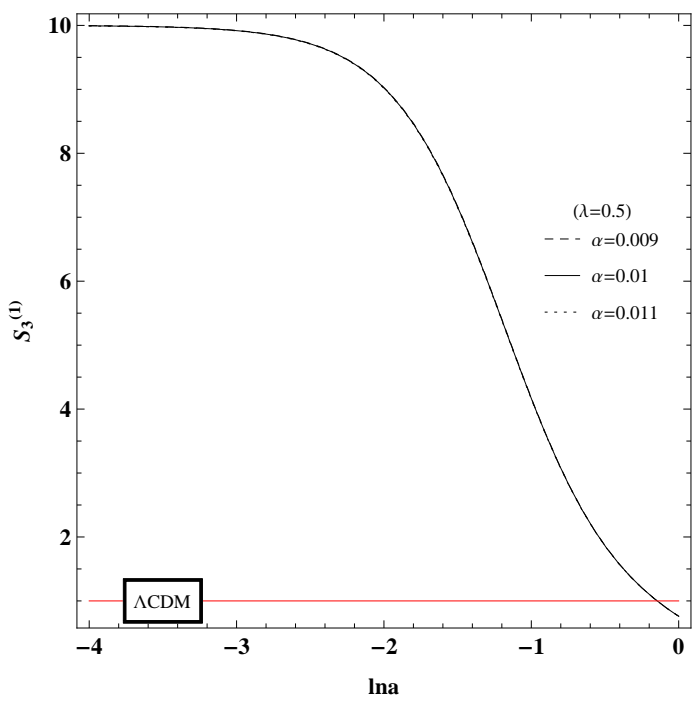

(a)

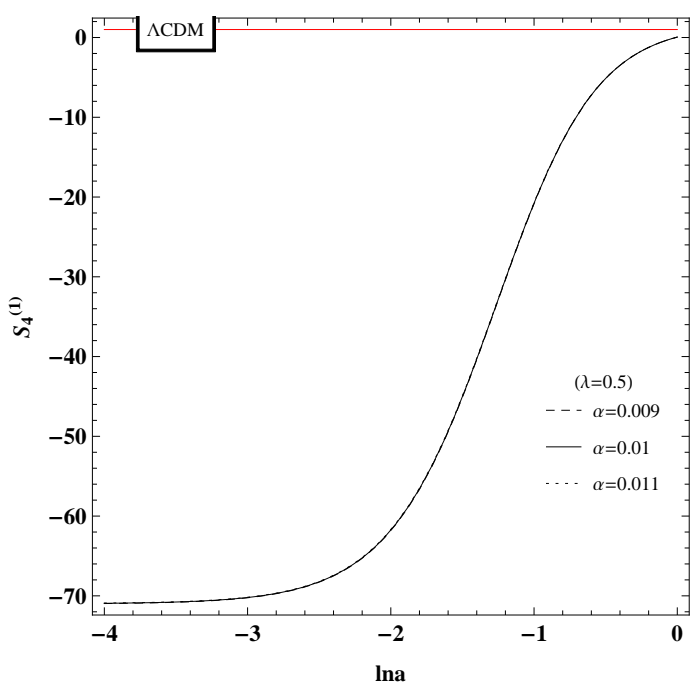

(b)

Figure 8. (a) Graph of $S_{3}^{(1)}$ for $P_{1}$ with different parameters $\alpha$ and fixed $\lambda=0.5$. (b) Graph of $S_{3}^{(1)}$ for $P_{1}$ with different parameters $\alpha$ and fixed $\lambda=0.5$. The initial point is $x_{0}=1, y_{0}=1.2$. 


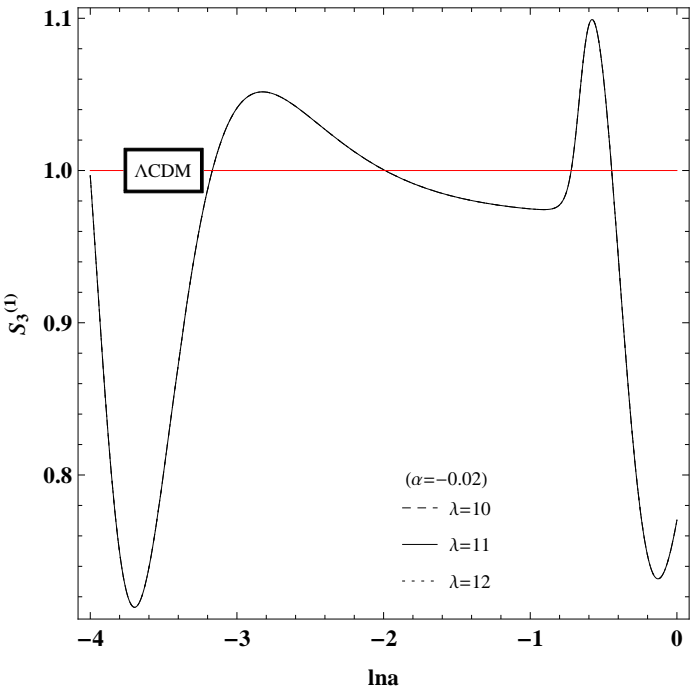

(a)

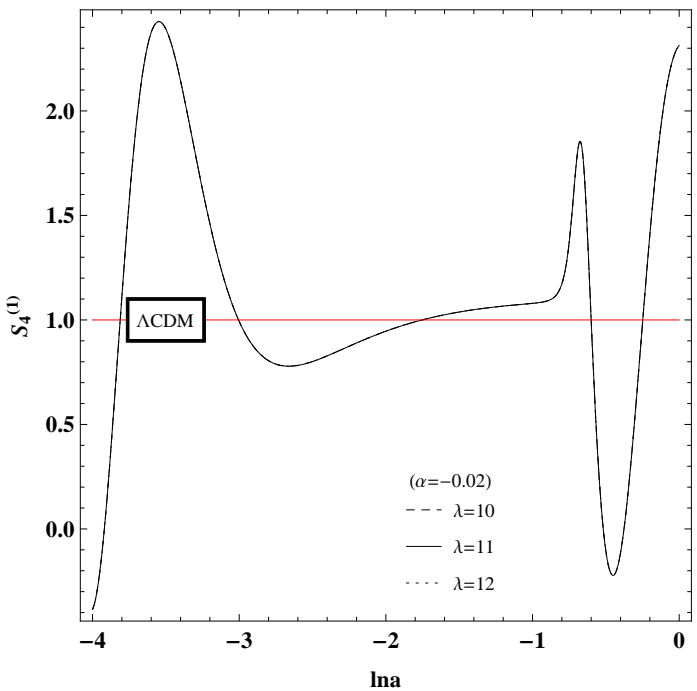

(b)

Figure 9. (a) Graph of $S_{3}^{(1)}$ for $P_{3}$ with different parameters $\lambda$ and fixed $\alpha=-0.02$. (b) Graph of $S_{4}^{(1)}$ for $P_{3}$ with different parameters $\lambda$ and fixed $\alpha=-0.02$. The initial point is $x_{0}=1, y_{0}=0.3$.

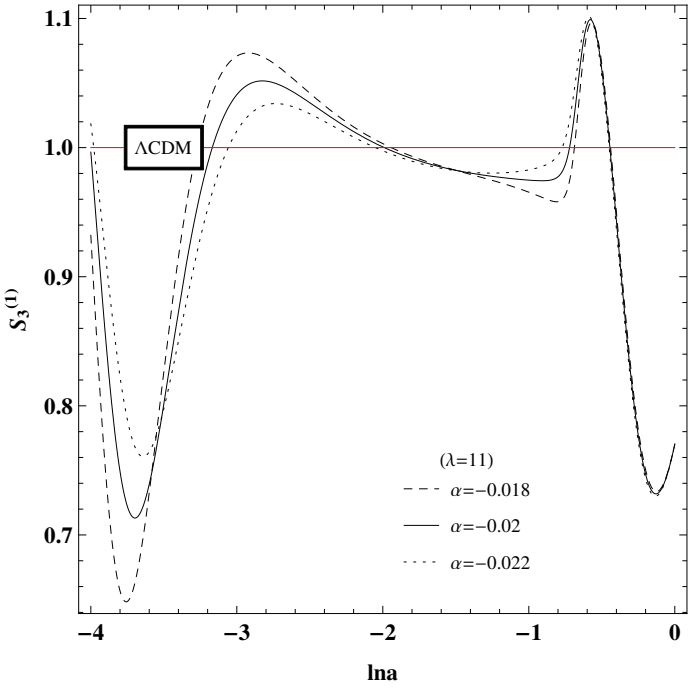

(a)

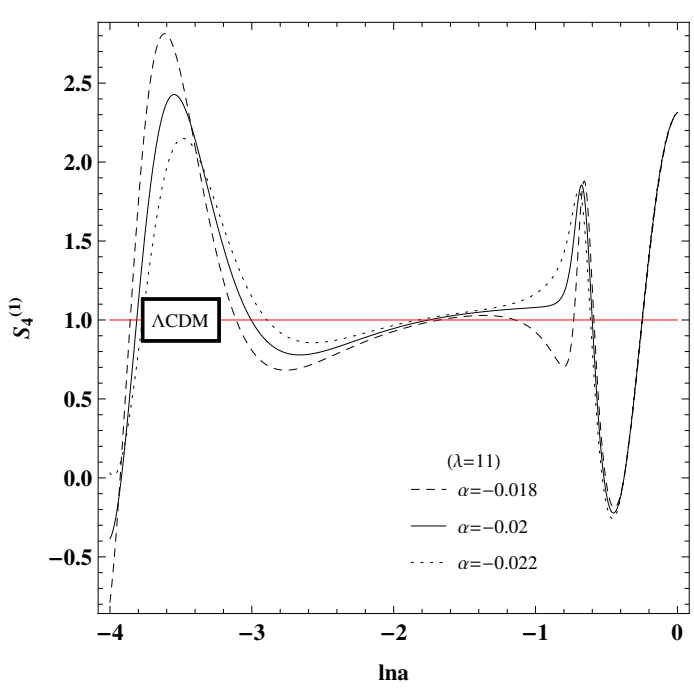

(b)

Figure 10. (a) Graph of $S_{3}^{(1)}$ for $P_{3}$ with different parameters $\alpha$ and fixed $\lambda=11$. (b) Graph of $S_{4}^{(1)}$ for $P_{3}$ with different parameters $\alpha$ and fixed $\lambda=11$. The initial point is $x_{0}=1, y_{0}=0.3$.

\section{Conclusions}

In summary, we have deeply investigated the cosmological evolution, the dynamical stability, as well as the geometrical diagnostic of the power law k-essence dark energy model with the Lagrangian containing a kinetic function $F(X)=-\sqrt{X}+X$ and interaction $Q=\alpha H \rho_{m}$ in FRWL space time. Concretely, we have not only discussed the influences of the coupling parameter $\alpha$ and potential parameter $\lambda$ on the evolution of several cosmological quantities (such as the density parameter $\Omega_{\phi}$, EoS of dark energy $w_{\phi}$, the effective sound speed $c_{s}^{2}$, and deceleration parameter $q$ ), but also numerically analyzed the dynamical stability and showed that there are the four dynamical attractors in the phase space. In addition, the statefinder hierarchy $S_{3}^{(1)}$ and $S_{4}^{(1)}$ of this dark energy model have been numerically obtained, which shows that the potential parameter $\lambda$ has more influence 
on the evolutions of $S_{3}^{(1)}$ and $S_{4}^{(1)}$ than one of the coupling parameter $\alpha$ around $P_{1}$ and $P_{4}$; while, for $P_{3}$ and $P_{6}$, parameter $\alpha$ plays a more important role in $S_{3}^{(1)}$ and $S_{4}^{(1)}$.

Author Contributions: B.-H.C.: search the references, plot the figures, write the manuscript; Y.-B.W.: corresponding author, review the manuscript, revise the manuscript; D.-F.X.: plot the figures, arrange the references, edit the manuscript; W.D.: plot the figures, explain the data, search the references; N.Z.: design the research plan, plot the figures, explain the data. All authors have read and agreed to the published version of the manuscript.

Funding: This research was funded by the National Natural Science Foundation of China grant number 12075109, 11575075,11705079 and 11865012.

Acknowledgments: Y. Wu has been supported by the National Natural Science Foundation of China under grants No.12075109 and No.11575075. W. Yang has been supported by the National Natural Science Foundation of China under grant No.11705079. J. Lu has been supported by the National Natural Science Foundation of China under grants No.11865012.

Conflicts of Interest: There is no conflict of interest.

\section{References}

1. Riess, A.G.; Filippenko, A.V.; Challis, P.; Clocchiatti, A.; Diercks, A.; Garnavich, P.M.; Gilliland, R.L.; Hogan, C.J.; Jha, S.; Kirshner, R.P.; et al. Observational Evidence from Supernovae for an Accelerating Universe and a Cosmological Constant. Astron. J. 1998, 116, 1009. [CrossRef]

2. Perlmutter, S.; Aldering, G.; Goldhaber, G.; Knop, R.A.; Nugent, P.; Castro, P.G.; Deustua, S.; Fabbro, S.; Goobar, A.; Groom, D.E.; et al. [The Supernova Cosmology Project] Measurements of $\Omega$ and $\Lambda$ from 42 High-Redshift Supernovae. Astrophys. J. 1999, 517, 565. [CrossRef]

3. Spergel, D.N.; Verde, L.; Peiris, H.V.; Komatsu, E.; Nolta, M.R.; Bennett, C.L.; Halpern, M.; Hinshaw, G.; Jarosik, N.; Kogut, A.; et al. First-year Wilkinson Microwave Anisotropy Probe (WMAP)* observations: Determination of cosmological parameters. Astrophys. J. Suppl. 2003, 148, 175. [CrossRef]

4. Eisenstein, D.J.; Zehavi, I.; Hogg, D.W.; Scoccimarro, R.; Blanton, M.R.; Nichol, R.C.; Scranton, R.; Seo, H.J.; Tegmark, M.; Zheng, Z.; et al. Detection of the baryon acoustic peak in the large-scale correlation function of SDSS luminous red galaxies. Astrophys. J. 2005, 633, 560. [CrossRef]

5. Aghanim, N.; Akrami, Y.; Ashdown, M.; Aumont, J.; Baccigalupi, C.; Ballardini, M.; Banday, A.J.; Barreiro, R.B.; Bartolo, N.; Basak, S.; et al. [Planck Collaboration] Planck 2018 results. VI. Cosmological parameters. Astron. Astrophys. J. 2020, 641, 1-67.

6. Xu, L.X.; Lu, J.B.; Wang, Y.T. Revisiting generalized Chaplygin gas as a unified dark matter and dark energy model. Eur. Phys. J. C 2012, 72, 1883. [CrossRef]

7. Xu, L.X.; Wang, Y.T.; Noh, H. Modified Chaplygin gas as a unified dark matter and dark energy model and cosmic constraints. Eur. Phys. J. C 2012, 72, 1931. [CrossRef]

8. Yang, W.Q.; Li, H.; Wu, Y.B.; Lu, J.B. Cosmological implications of the dark matter equation of state. Int. J. Mod. Phys. D 2017, 26, 1750013. [CrossRef]

9. Du, M.H.; Yang, W.Q.; Xu, L.X.; Pan, S.; Mota, D.F. Future constraints on dynamical dark-energy using gravitational-wave standard sirens. Phys. Rev. D 2019, 100, 043535. [CrossRef]

10. Sahni, V.; Starobinsky, A.A. The case for a positive cosmological $\Lambda$-term. Int. J. Mod. Phys. D 2000, 9, 373. [CrossRef]

11. Carroll, S.M. The cosmological constant. Living Rev. Rel. 2001, 4, 1. [CrossRef] [PubMed]

12. Carroll, S.M.; Hoffman, M.; Trodden, M. Can the dark energy equation-of-state parameter w be less than -1 ? Phys. Rev. D 2003, 68, 023509. [CrossRef]

13. Li, M.; Li, X.D.; Wang, S.; Wang, Y. Commun. Dark Energy. Theor. Phys. 2011, 56, 525-604.

14. Yang, R.J.; Zhang, S.N. The age problem in the $\Lambda$ CDM model. Mon. Not. R. Astron. Soc. 2010, 407, $1835-1841$. [CrossRef]

15. Valentino, E.D.; Melchiorri, A.; Silk, J. Reconciling Planck with the local value of $H_{0}$ in extended parameter space. Phys. Lett. B 2016, 761, 242. [CrossRef]

16. Huang, Q.G.; Wang, K. How the dark energy can reconcile Planck with local determination of the Hubble constant. Eur. Phys. J. C 2016, 76, 506. [CrossRef] 
17. Vagnozzi, S. New physics in light of the $H_{0}$ tension: An alternative view. Phys. Rev. D 2020, 102, 023518. [CrossRef]

18. Kumar, S.; Nunes, R.C. Probing the interaction between dark matter and dark energy in the presence of massive neutrinos. Phys. Rev. D 2016, 94, 123511. [CrossRef]

19. Pan, S.; Yang, W.Q.; Singha, C.; Saridakis, E.N. Observational constraints on sign-changeable interaction models and alleviation of the $H_{0}$ tension. Phys. Rev. D 2019, 100, 083539. [CrossRef]

20. Pan, S.; Yang, W.Q.; Valentino, E.D.; Saridakis, E.N.; Chakraborty, S. Interacting scenarios with dynamical dark energy: Observational constraints and alleviation of the $H_{0}$ tension. Phys. Rev. D 2019, 100, 103520. [CrossRef]

21. Tsujikawa, S. Quintessence: A Review. Class. Quant. Grav. 2013, 30, 214003. [CrossRef]

22. Feng, B.; Wang, X.L.; Zhang, X.M. Dark Energy Constraints from the Cosmic Age and Supernova. Phys. Lett. B 2005, 607, 35-41. [CrossRef]

23. Armendariz-Picon, C.; Damour, T.; Mukhanov, V. K-inflation. Phys. Lett. B 1999, 458, 209-218. [CrossRef]

24. Armendariz-Picon, C.; Mukhanov, V.; Steinhardt, P.J. A Dynamical Solution to the Problem of a Small Cosmological Constant and Late-time Cosmic Acceleration. Phys. Rev. Lett. 2000, 85, 4438-4441. [CrossRef]

25. Rendall, A.D. Dynamics of k-essence. Class. Quant. Grav. 2006, 23, 1557-1570. [CrossRef]

26. Scherrer, R.J. Purely kinetic k-essence as unified dark matter. Phys. Rev. Lett. 2004, 93, 011301. [CrossRef]

27. Bose, N.; Majumdar, A.S. A k-essence Model of Inflation, Dark Matter and Dark Energy. Phys. Rev. D 2009, 79, 103517. [CrossRef]

28. Bose, N.; Majumdar, A.S. Unified Model of k-Inflation, Dark Matter and Dark Energy. Phys. Rev. D 2009, 80, 103508. [CrossRef]

29. Chiba, T.; Okabe, T.; Yamaguchi, M. Kinetically Driven Quintessence. Phys. Rev. D 2000, 62, 023511. [CrossRef]

30. Chimento, L.P.; Feinstein, A. Power-law expansion in k-essence cosmology. Mod. Phys. Lett. A 2004, 19, 761-768. [CrossRef]

31. Chimento, L.P. Extended tachyon field, Chaplygin gas and solvable k-essence cosmologies. Phys. Rev. D 2004, 69, 123517. [CrossRef]

32. Battye, R.A.; Pace, F. Approximation of the potential in scalar field dark energy models. Phys. Rev. D 2016, 94, 063513. [CrossRef]

33. Wang, B. Dark Matter and Dark Energy Interactions: Theoretical Challenges, Cosmological Implications and Observational Signatures. Rep. Prog. Phys. 2016, 79, 096901. [CrossRef] [PubMed]

34. Chimento, L.P. Linear and nonlinear interactions in the dark sector. Phys. Rev. D. 2010, 81, 043525. [CrossRef]

35. Paliathanasis, A.; Pan, S.; Yang, W.Q. Dynamics of nonlinear interacting dark energy models. Int. J. Mod. Phys. D 2019, 28, 1950161 [CrossRef]

36. Yang, W.Q.; Pan, S.; Barrow, J.D. Large-scale stability and astronomical constraints for coupled dark-energy models. Phys. Rev. D. 2018, 97, 043529. [CrossRef]

37. zhang, N.; Wu, Y.B.; Chi, J.N.; Yu, Z.; Xu, D.F. Diagnosing Tsallis holographic dark energy models with interactions. Mod. Phys. Lett. A 2020, 33, 2050044 [CrossRef]

38. Yang, R.J.; Chen, B.H.; Li, J.; Qi, J.Z. The evolution of the power law k-essence cosmology. Astrophys. Space Sci. 2015, 356, 399-405. [CrossRef]

39. Copeland, E.J.; Sami, M.; Tsujikawa, S. Dynamics of dark energy. Int. J. Mod. Phys. D. 2006, 15, $1753-1935$. [CrossRef]

40. Bahamonde, S.; Boehmer, C.G.; Carloni, S.; Copeland, E.J.; Fang, W.; Tamanini, N. Dynamical systems applied to cosmology: Dark energy and modified gravity. Phys. Rep. 2018, 775, 1-122. [CrossRef]

41. Leon, G.; Saridakis, E.N. Phase-space analysis of Horava-Lifshitz cosmology. J. Cosmol. Astropart. Phys. 2009, 2009, 006. [CrossRef]

42. Sahni, V.; Shafieloo, A.; Starobinsky, A.A. Two new diagnostics of dark energy. Phys. Rev. D 2008, 78, 103502. [CrossRef]

43. Wu, Y.B.; Zhang, C.Y.; Lu, J.B.; Lu, J.W.; Zhang, X.; Qi, Q.M.; Fan, B. Analysis on modified Chaplygin gas as dark energy model. Mod. Phys. Lett. A 2015, 30, 1550005. [CrossRef]

44. Sahni, V.; Saini, T.D.; Starobinsky, A.A.; Alam, U. Statefinder-A new geometrical diagnostic of dark energy. JETP Lett. 2003, 77, 201-206. [CrossRef] 
45. Alam, U.; Sahni, V.; Saini, T.D.; Starobinsky, A.A. Exploring the Expanding Universe and Dark Energy using the Statefinder Diagnostic. Mon. Not. R. Asron. Soc. 2003, 344, 1057. [CrossRef]

46. Arabsalmani, M.; Sahni, V. The Statefinder hierarchy: An extended null diagnostic for concordance cosmology. Phy. Rev. D. 2011, 83, 043501. [CrossRef]

47. Li, J.; Yang, R.J.; Chen, B.H. Discriminating dark energy models by using the statefinder hierarchy and the growth rate of matter perturbations. J. Cosmol. Astropart. Phys. 2014, 2014, 043. [CrossRef]

48. Cui, J.L.; Yin, L.; Wang, L.F.; Li, Y.H.; Zhang, X. A closer look at interacting dark energy with statefinder hierarchy and growth rate of structure. J. Cosmol. Astropart. Phys. 2014, 2014, 024. [CrossRef]

49. Liu, W.Z.; Liu, D.J. Statefinder Diagnostic for Quintessence with or without Thermal Interaction. Int. J. Mod. Phys. D 2009, 18, 43-52. [CrossRef]

50. De-Santiago, J.; Cervantes-Cota, J.L. Generalizing a Unified Model of Dark Matter, Dark Energy, and Inflation with Non Canonical Kinetic Term. Phys. Rev. D 2011, 83, 063502. [CrossRef]

51. Garriga, J.; Mukhanov, V.F. Perturbations in k-inflation. Phys. Lett. B 1999, 458, 219-225. [CrossRef]

52. Unnikrishnan, S.; Sriramkumar, L. A note on perfect scalar fields. Phys. Rev. D 2010, 81, 103511. [CrossRef]

53. Christopherson, A.J.; Malik, K.A. The non-adiabatic pressure in general scalar field system. Phys. Lett. B 2009, 675, 159-163. [CrossRef]

54. Copeland, E.J.; Liddle, A.R.; Wands, D. Exponential potentials and cosmological scaling solutions. Phys. Rev. D 1998, 57, 4686-4690. [CrossRef]

55. Yang, R.J.; Gao, X.T. Phase-space analysis of a class of k-essence cosmology. Class. Quantum Gravity 2011, 28, 065012. [CrossRef]

56. Capozziello, S.; Nojiri, S.; Odintsov, S.D. Unified phantom cosmology: Inflation, dark energy and dark matter under the same standard. Phys. Lett. B 2006, 632, 597-604. [CrossRef]

57. Carloni, S.; Elizalde, E.; Silva, P.J. An analysis of the phase space of Horava-Lifshitz cosmologies. Class. Quant. Grav. 2010, 27, 045004. [CrossRef]

58. He, J.; Wu, Y.B.; Fu, M.H. Dynamical Attractor of Modified Chaplygin Gas. Chin. Phys. Lett. 2008, 25, 347.

Publisher's Note: MDPI stays neutral with regard to jurisdictional claims in published maps and institutional affiliations.

(C) 2020 by the authors. Licensee MDPI, Basel, Switzerland. This article is an open access article distributed under the terms and conditions of the Creative Commons Attribution (CC BY) license (http:/ / creativecommons.org/licenses/by/4.0/). 